\title{
La salud mental en el paciente con obesidad en protocolo para cirugía bariátrica
}

\author{
Héctor Esquivias-Zavala, ${ }^{1}$ Aída Monserrat Reséndiz-Barragán, ${ }^{1}$ Fernando García, ${ }^{2}$ Daniel Elías-López ${ }^{3}$
}

Artículo de revisión

\section{ABSTRACT}

\section{Background}

The association between obesity and psychopathology is well known. However, the presence of psychopathology in patients with morbid obesity who attend bariatric surgery and its impact in weight loss has been less studied.

\section{Objective}

To perform a theme update about the general context of psychiatric disorders associated with morbid obesity, and to assess the impact of such disorders in patients undergoing bariatric surgery.

\section{Method}

EBSCO Research Database and PubMed databases were consulted. Articles published between 2011 and today were selected. Key words related to the topic of study were used.

\section{Results}

The studies reviewed showed five psychopathological clinical entities with high prevalence in patients who attend bariatric surgery: Depression and anxiety, attention deficit hyperactivity disorder, disorders of eating behavior, substance abuse, and some dimensions of personality. The presence of these disorders was associated to a poor post-surgical weight loss.

\section{Discussion and conclusion}

Binge eating disorder and substance abuse impact on weight loss after bariatric surgery is controversial. The evaluation of obese patients who attend bariatric surgery must emphasize, according with reported disorders prevalence, the identification of depression, anxiety and attention deficit hyperactivity disorder.

Key words: Mental health, morbid obesity, bariatric surgery, weight loss, psychopathology, depressive disorder.

\section{RESUMEN}

\section{Antecedentes}

La asociación entre obesidad y psicopatología es bien conocida; sin embargo, la presencia de esta última en los pacientes con obesidad mórbida sometidos a cirugía bariátrica y su impacto en la pérdida de peso ha sido menos estudiado.

\section{Objetivo}

Realizar una actualización sobre el panorama general de los trastornos psiquiátricos asociados con la obesidad mórbida, y el impacto de éstos sobre la pérdida de peso en pacientes bariátricos.

\section{Método}

Se consultaron las bases de datos EBSCO Research Database y PubMed. Se seleccionaron los artículos publicados de 2011 a la fecha. Fueron utilizadas palabras clave relacionadas con el tema de estudio.

\section{Resultados}

Los estudios revisados mostraron cinco entidades clínicas psicopatológicas con alta prevalencia en pacientes que se encuentran en protocolo de cirugía bariátrica: Depresión y ansiedad, trastorno por déficit de atención e hiperactividad, trastornos de la conducta alimentaria, abuso de sustancias y algunas dimensiones de la personalidad. La presencia de estas condiciones se asoció a una menor pérdida de peso posterior al procedimiento quirúrgico.

\section{Discusión y conclusión}

El impacto del trastorno por atracón y por abuso de sustancias en la pérdida de peso, posterior a la cirugía bariátrica, es controversial. La evaluación del paciente con obesidad candidato a cirugía bariátrica debe enfatizar, de acuerdo con la prevalencia de los trastornos reportados, la identificación de trastornos depresivos-ansiosos y del trastorno por déficit de atención e hiperactividad.

Palabras clave: Salud mental, obesidad mórbida, cirugía bariátrica, pérdida de peso, psicopatología, trastorno depresivo.

Clínica de Obesidad y Control de Peso, Hospital General Dr. Manuel Gea González, México.

2 Centro de Atención Integral a la Salud Mental de León, Guanajuato, México.

Departamento de Endocrinología y Metabolismo, Instituto Nacional de Ciencias Médicas y Nutrición Salvador Zubirán.

Correspondencia: Héctor Esquivias-Zavala, Clínica de Obesidad y Control de Peso, Hospital General Dr. Manuel Gea González, Av. Calz. de Tlalpan 4800, Sección XVI, Tlalpan, 14080, Ciudad de México, México. Tel: (55) 4000 - 3000 ext. 3687. E-mail: doctoresquivias@hotmail.com

Recibido primera versión: 7 de abril de 2016. Aceptado: 6 de mayo de 2016. 


\section{INTRODUCCIÓN}

La obesidad es una epidemia mundial que afecta la calidad de vida de quienes la padecen, aumenta el riesgo de adquirir enfermedades y eleva los costos del cuidado en salud en prácticamente todos los países del mundo. ${ }^{1}$ La naturaleza del padecimiento es generada por la interacción entre diversos factores: genéticos, epigenéticos, fisiológicos, conductuales, socioculturales y ambientales. Esto lleva a un desequilibrio, donde coexiste una mayor ingesta de energía y un menor gasto calórico por un tiempo prolongado. ${ }^{2}$

En México, las encuestas demuestran que actualmente la obesidad ha desplazado a la desnutrición como principal reto por resolver. ${ }^{3}$ Los datos de la Encuesta Nacional de Salud y Nutrición 2012 reportaron que la prevalencia de sobrepeso y obesidad en adultos mexicanos de 20 años o más fue de $71.3 \%$ (obesidad $32.4 \%$ y sobrepeso $38.8 \%$ ), reportaron además que la obesidad es más alta en el sexo femenino (37.5\% vs. $26.8 \%) .{ }^{4}$

$\mathrm{El}$ incremento progresivo en la prevalencia de obesidad obliga al desarrollo de estrategias efectivas para afrontarla. Las medidas preventivas implementadas en las escuelas, lugares de trabajo y en la comunidad han tenido un efecto menor, ${ }^{5}$ por lo que en la mayoría de los casos está indicado el tratamiento que incluye cambios en el estilo de vida, modificaciones en la alimentación, aumento en la actividad física, uso de la terapia farmacológica y, en algunos pacientes, el manejo quirúrgico.

El principal reto por vencer en el tratamiento de la obesidad es mantener la pérdida de peso por un tiempo prolongado. El tratamiento conductual y farmacológico ha demostrado en diversos estudios ser efectivo, pero un alto porcentaje recupera el peso de forma gradual., ${ }^{6,7}$ El tratamiento conservador, basado en cambios en el estilo de vida e intervenciones farmacológicas en pacientes con índice de masa corporal $(\mathrm{IMC})>35 \mathrm{Kg} / \mathrm{m}^{2}$ es inefectivo en cuanto a pérdida ponderal y como tratamiento de los fenómenos comórbidos. Sin embargo, la cirugía bariátrica (CB) ha demostrado mayor efectividad en el tratamiento de estas variables a largo plazo. ${ }^{8}$ En 1991, los Institutos Nacionales de Salud de los Estados Unidos de América emitieron una serie de recomendaciones relacionadas con el perfil que deben cumplir los pacientes con obesidad mórbida para ser candidatos a $\mathrm{CB}$, dichas recomendaciones incluyen a los pacientes con IMC $>40 \mathrm{Kg} / \mathrm{m}^{2}$ o $35 \mathrm{Kg} / \mathrm{m}^{2}$ con alguna de las enfermedades comórbidas a la obesidad. Lo anterior, derivado de la estimación de que por cada $5 \mathrm{Kg} / \mathrm{m}^{2}$ de incremento en el IMC, sobre una base de $25 \mathrm{Kg} / \mathrm{m}^{2}$ existe un aumento paulatino en la mortalidad general, de manera que al llegar a los 30$35 \mathrm{Kg} / \mathrm{m}^{2}$ la mediana de supervivencia se reduce en 2-4 años y al llegar al IMC de 40-45 Kg/m² se reduce en 8-10 años. ${ }^{9}$

Hacia 2003, en México se realizaban 2500 procedimientos bariátricos al año. ${ }^{10}$ La selección adecuada de los pacientes es fundamental para alcanzar los objetivos deseados y mantener un cambio permanente. El proceso requiere una evaluación integral y meticulosa llevada a cabo por un equipo multidisciplinario que incluye una evaluación médica, nutricional, deportiva, psiquiátrica y psicológica profunda. Esta última evalúa la conciencia del paciente sobre su condición y sus expectativas de la cirugía, debe demostrar, además, capacidad y voluntad para modificar sus hábitos y su estilo de vida. Estos aspectos son fundamentales durante el postoperatorio y para el éxito de la intervención.

La psicopatología es un factor asociado con la obesidad y aunque no se ha establecido claramente si es causa o efecto de ésta, sí se conoce la interacción entre los síntomas emocionales y su impacto en la desestructuración de los patrones de alimentación y el sedentarismo, ambos factores identificados y asociados con la obesidad.

Varios estudios han señalado la presencia de abuso físico y sexual en los pacientes con obesidad, así como una prevalencia de trastornos psiquiátricos entre $30-60 \%$, reconociendo los trastornos afectivos, los de ansiedad y los trastornos de la conducta alimentaria (TCA) como los más frecuentes. ${ }^{11-13}$ En relación con el abuso físico o sexual, distintos estudios identificaron la asociación entre maltrato y abuso sexual en la infancia con el riesgo de incrementar la obesidad en relación con el número y las formas de abuso; ${ }^{14,15}$ razón por la cual algunos autores describieron la obesidad como una función adaptativa de "des-sexualizarse" como una forma de protección.

Durante el abordaje prequirúrgico multidisciplinario, los trastornos psicopatológicos son identificados por los profesionistas en salud mental. Existen trastornos específicos como el trastorno depresivo mayor grave, los trastornos de la personalidad, los trastornos psicóticos, los trastornos de aprendizaje graves y el abuso de alcohol y drogas, cuya presencia se asocia con una menor pérdida de peso después de la CB. Fundamentados en lo anterior, los grandes grupos académicos han señalado a estas entidades como una contraindicación para el tratamiento quirúrgico para la obesidad. ${ }^{16-20}$

Por lo anterior, y en función de una buena práctica clínica, es relevante realizar esta actualización sobre las principales entidades psicopatológicas comórbidas a los pacientes bariátricos, para lograr una selección adecuada de los candidatos idóneos.

\section{OBJETIVO}

Realizar una actualización del panorama general de los trastornos psiquiátricos, su asociación con la obesidad y el impacto de éstos en la pérdida de peso de los pacientes bariátricos.

\section{MÉTODO}

Para la integración de esta revisión se consultaron las bases de datos EBSCO Research Database, y PubMed; se realizó una 
búsqueda en español e inglés de artículos originales, publicados del 2011 a la fecha, excepto para las publicaciones clásicas sobre el tema. Las palabras clave utilizadas para la búsqueda fueron: "bariatric surgery" "and" los siguientes términos: "attention deficit hyperactivity disorder", "anxiety", "depression", "eating disorders", "personality disorders", "substance abuse" y "mental health". Se identificaron un total de 69 artículos de los cuáles se eliminaron tres por no presentar información relevante sobre el tema. Del total de artículos revisados, fueron incluidos 66 en función de su importancia para cumplir con el objetivo del manuscrito, se identificaron 20 revisiones narrativas, 33 estudios clínicos (longitudinales, prospectivos, casos clínicos), nueve reportes epidemiológicos y cuatro revisiones sistemáticas. Se consultaron además las guías de práctica clínica internacionales y los documentos para el manejo de la obesidad en México emitidos por expertos en políticas públicas de la Universidad Nacional Autónoma de México y la Academia Nacional de Medicina.

Los resultados fueron agrupados en cinco entidades clínicas, que se presentan de acuerdo con la prevalencia de los trastornos agrupados en cada una de éstas. Se reportan las manifestaciones clínicas de cada trastorno en la población bariátrica y su impacto en la pérdida de peso.

\section{RESULTADOS}

\section{Depresión y ansiedad}

En México, el trastorno depresivo (TD) es uno de los más frecuentes de acuerdo con la Encuesta Nacional de Epidemiología Psiquiátrica (2003) donde los trastornos afectivos se presentaron en el 9.1\% de la población. ${ }^{21}$ En cambio, en población obesa se ha reportado en un 30\%, es decir en tres de cada 10 pacientes y al igual que en la población general, con mayor frecuencia en el sexo femenino. ${ }^{22,23}$

La asociación TD-obesidad surgió de la comorbilidad con el trastorno por atracón (TPA) observada en los pacientes obesos. La mayor intensidad del TD en pacientes obesos con atracón, a diferencia de aquellos obesos sin atracón, fue lo que postuló la hipótesis de la asociación TD-obesidad. ${ }^{23}$

El trastorno de ansiedad se presenta hasta en un 35\% de los pacientes con obesidad y se ha reportado que con frecuencia coexiste con la depresión. ${ }^{22,23}$ Aunque el vínculo ansiedad-obesidad ha sido menos estudiado que depresión-obesidad, una explicación para éste es el fenómeno descrito como "ingesta emocional" derivado de un pobre autocontrol y de una limitación para contender con situaciones estresantes, siendo la conducta alimentaria mediadora entre estas condiciones. ${ }^{23,24}$

Un estudio encontró como factores asociados a estos trastornos, haber tenido o iniciado la obesidad en la infancia y haber presentado cambios en el IMC a lo largo de la vida. ${ }^{25}$ Otros estudios reportaron la asociación del TD con una mayor frecuencia de estigmatización debida al peso corporal. ${ }^{25,26} \mathrm{Y}$ otros, identificaron el antecedente de violencia sexual o física y de otras situaciones de desventaja social y laboral como factores asociados. ${ }^{14,15}$

El TD en los pacientes con sobrepeso y obesidad se caracteriza por un mayor número de recaídas y un importante componente de desesperanza, por lo que un estudio concluyó que la depresión en ellos es más severa. ${ }^{27}$ Aunque se ha documentado que a mayor IMC el riesgo de suicido es menor, éste se encuentra presente en el $4 \%$ de los pacientes sometidos a CB. ${ }^{28}$ Diversos estudios han mostrado una alta prevalencia de suicidio después de la cirugía ${ }^{29}$ y otros han asociado este incremento a la recaída en el consumo de alcohol $^{30,31}$ y a la exacerbación de los síntomas depresivos. ${ }^{32}$ Una posible hipótesis para estos fenómenos es la modificación en las vías de señalización de la ghrelina, hormona reguladora del metabolismo energético pero que también regula las respuestas de ansiedad y depresión, tiene un papel en el funcionamiento de la memoria y es neuroprotectora en condiciones degenerativas e isquémicas. ${ }^{33}$

Un estudio $(N=531)$ realizado en tres grupos de pacientes obesos, dos de ellos con una intervención para pérdida de peso (tratamiento convencional y tratamiento quirúrgico) y el tercer grupo control sin ninguna intervención, reportó que los trastornos depresivos-ansiosos influyeron negativamente en la pérdida de peso en los grupos de intervención e incluso, el grupo control con estas comorbilidades presentó un incremento de peso. Al comparar la reducción del peso entre los pacientes con y sin estas comorbilidades, se demuestra que la presencia de estos trastornos influye negativamente en el éxito de estas intervenciones. ${ }^{23}$

\section{Trastorno por déficit de atención e hiperactividad (TDAH)}

Este trastorno tiene una prevalencia en la población general del 3-4\%. Aunque generalmente es diagnosticado en escolares (prevalencia 5-10\%), de éstos aprox. el 60\% persiste con síntomas durante la edad adulta. ${ }^{34}$

La primera asociación TDAH-obesidad se reportó en $2002^{35}$ y un estudio en adultos con diagnóstico retrospectivo de TDAH en la infancia confirmó esta asociación. ${ }^{36}$ Su prevalencia en la población obesa es del 27.4\% (aunque la manifestación subsindromática podría estar presente en el 33\%) y se ha identificado en el $10.2 \%$ de los candidatos a CB. ${ }^{35}$

El vínculo TDAH-obesidad sugiere una regulación genética que está mediada por una asociación entre una mutación del gen que codifica la proteína MC4-R, presente en pacientes con TDAH y que se localiza en el exón único $18 \mathrm{q}$ del cromosoma 22, cuyo déficit se ha asociado también con las vías neuronales que regulan el hambre y la saciedad. ${ }^{37-39}$

El mecanismo neurobiológico de esta asociación es la disfunción en el control inhibitorio, que altera el funciona- 
miento ejecutivo generando distraibilidad, desorganización e incumplimiento en hábitos de vida saludables. ${ }^{40}$ Además, los pacientes con TDAH muestran menos atención a las señales internas de hambre y saciedad, ${ }^{41} \mathrm{y}$ asociado con un componente impulsivo, presentan con frecuencia abuso de sustancias ${ }^{42,43}$ y además, pobre tolerancia a la espera que genera mayor ingesta de alimentos hipercalóricos. ${ }^{44}$ Se ha reportado que, cuando coexiste TDAH-obesidad, la probabilidad de presentar atracones incrementa 3.97 veces más que en los obesos sin TDAH. ${ }^{45,46}$ Actualmente, las guías de la American Society for Metabolic and Bariatric Surgery puntualizan en evaluar la impulsividad como un factor asociado con menor pérdida de peso y mayores complicaciones

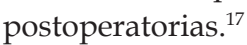

El TDAH favorece en este grupo una pobre capacidad para adherirse a patrones regulares de alimentación, ${ }^{47}$ el cual presenta mayor dificultad para cumplir los objetivos del protocolo prequirúrgico y menor adherencia al tratamiento y seguimiento médico. ${ }^{48}$ Esto podría explicar la mayor recidiva de obesidad después de la CB. ${ }^{49}$

Además de la asociación entre el TDAH y el TPA, se ha demostrado que éste se presenta también con otros síndromes de la conducta alimentaria como el comedor nocturno y la ingesta emocional..$^{50}$ Se han reportado asociaciones significativas entre TDAH-TD y TDAH-ansiedad entre los obesos. ${ }^{51,52}$ Estas comorbilidades complican el abordaje y representan un reto mayor en su tratamiento.

La intervención farmacológica del TDAH impactó de forma significativa al comparar el peso entre los pacientes que recibieron tratamiento con los que no lo recibieron, pérdida de $10.35 \%$ en los tratados y una ganancia del $7.03 \%$ en los controles $(p<0.001){ }^{36,53}$

\section{Trastornos de la conducta alimentaria (TCA)}

Actualmente la obesidad no es considera en el grupo de los TCA y, aunque se ha sugerido incluirla como tal, éstos presentan elementos psicopatológicos que no están presentes en la obesidad, como la sobrevaloración de la imagen corporal, distinta a la insatisfacción con la imagen corporal (IIC). Por otro lado, a diferencia de los trastornos por abuso de sustancias, el comer es regulado por factores homeostásicos periféricos (leptina, ghrelina, insulina), por lo que es debatible incluir la obesidad en el campo de las adicciones. Finalmente, a diferencia de éstas el consumo de alimentos es esencial para la vida. ${ }^{54,55}$

Un estudio en adolescentes mexicanos $(N=25056)$, encontró que aquellos con sobrepeso y obesidad, comparados con pares de peso normal, presentaron más posibilidades de desarrollar conductas alimentarias de riesgo en ambas categorías (O.R. 2.76, IC 95\%, 2.17-3.51 y O.R. 4.58, IC 95\%, 3.47-6.07) respectivamente..$^{56}$

La prevalencia del TPA en población general es entre 2-5\%; sin embargo, en la población obesa, esta conducta fue la más reportada (27\%) y se incrementó de forma proporcional al IMC..$^{22,57}$ El TPA se reportó en el 25\% de las personas con sobrepeso que buscan tratamiento para perderlo. ${ }^{22}$

Una revisión realizada con el objetivo de explorar el impacto del TPA en los cambios ponderales, la conducta alimentaria y los resultados postquirúrgicos, encontró una amplia varianza en la prevalencia desde $6 \%$ y hasta $64 \% .{ }^{58}$ Un estudio mostró que cuatro de cada nueve sujetos que se atracaban antes de la cirugía continuaban haciéndolo después. Otro estudio de seguimiento, un año después de la $\mathrm{CB}$, reportó que el $24.2 \%$ de los pacientes persistían con esta conducta. ${ }^{59,60}$ Un estudio retrospectivo reportó que 14 de 38 pacientes que recordaron haber presentado atracones antes de la cirugía continuaban presentándolos hasta 15 años después. La conducta se presentó principalmente en aquellos que ya tenían atracones previos y $4.5 \%$ los reportaron de novo. ${ }^{61}$ Otras conductas como el grazing (consumo de alimentos por periodos prolongados durante el día, por pérdida del control), fueron reiniciadas en el $80 \%$ de los sujetos seis meses después de la cirugía; muchos de ellos pese a identificar su conducta como un atracón, no cumplieron los criterios del DSM-IV por sentirse limitados en la cantidad de alimentos consumidos. ${ }^{62}$ Varios estudios en pacientes con TPA reportaron mayor riesgo de incremento ponderal dos y hasta siete años después de la cirugía. ${ }^{63}$ Se encontró un estudio que no reportó diferencias en el peso entre pacientes con y sin TPA. Sin embargo, este trabajó proporcionó un tratamiento específico a los pacientes con este diagnóstico. ${ }^{64}$

Un estudio $(N=195)$ encontró que los sujetos con TPA reportaron mayor psicopatología en comparación con los que no lo tenían $(p=0.001)$, además reportaron un mayor porcentaje de trastornos psiquiátricos a lo largo de la vida $(p=0.007)$ y mayor frecuencia de antecedentes como trastornos afectivos $(p=0.003)$ y ansiosos $(p=0.019)$. Los diagnósticos más frecuentes para los grupos con y sin atracón fueron el trastorno depresivo mayor y el trastorno de pánico sin agorafobia. El antecedente de abuso sexual se reportó en el $22.2 \%$ de los sujetos con TPA y en el $7.1 \%$ entre quienes no tenían este diagnóstico, aproximándose a la significancia estadística $(p=0.06) .{ }^{65}$

Un estudio encontró que las mujeres candidatas a $\mathrm{CB}$ reportaron mayores puntuaciones en IIC, en tanto, los hombres reportaron mayores IMC. La IIC se correlacionó con mayor frecuencia de atracones, más síntomas de ansiedad y menor autoestima. ${ }^{54}$

La asociación entre el TPA-IIC, se explica mediante la restricción alimentaria que produce hambre y posteriormente comer en exceso. Otros factores reportados en asociación con el TPA fueron tener obesidad, iniciar dietas a edades más tempranas y el mayor número de intentos de pérdida de peso. ${ }^{66,67}$

Existe un reporte que identificó una serie de casos después de la $\mathrm{CB}$ como: restricción alimentaria intensa, temor intenso a subir de peso, TPA, sobrevaloración de la silueta 
corporal o del peso en la autoevaluación, vómito, ejercicio excesivo, abuso de laxantes y diuréticos, entre otras conductas o síntomas. ${ }^{68}$ Un estudio de seguimiento, que exploró la asociación entre la conducta alimentaria y el porcentaje de peso excesivo perdido, la recuperación de peso y la calidad de vida percibida, reportó que el $49.9 \%$ de los sujetos presentaron pérdida de control al comer, $27 \%$ manifestaron mayor ingesta a la esperada para una persona post-CB y el $18 \%$ reportó criterios completos para diagnóstico de TPA de acuerdo con el DSM-IV. Se reportaron asociaciones entre la pérdida de control al comer y el porcentaje de peso excesivo perdido y recuperación ponderal $(p<0.001)$ y $(p=0.013)$ respectivamente, así como entre la frecuencia de atracones y recuperación ponderal $(P=0.006)$. El $46.6 \%$ reportaron conducta de "pica", su frecuencia se correlacionó negativamente con el porcentaje de peso perdido $(p<0.001)$ y positivamente con la recuperación corporal $(p<0.001)$. Los participantes con TPA reportaron una pobre calidad de vida $(p<0.001)$ similar a quienes reportaron frecuencia de "pica" mayor a dos veces por semana $(p<0.001)$. Aquellos que recuperaron $>10 \%$ del peso perdido después de la CB habían presentado mayor frecuencia de atracones $(p=0.03)$, "pica" $(p<0.001)$, pérdida de control $(p<0.001)$ y peor calidad de vida $(p<0.001){ }^{69}$

Un estudio realizado en postquirúrgicos de $\mathrm{CB}$ con y sin TPA comparados con grupos control (sin CB), evaluó si los sujetos con TPA perderían significativamente más peso 12 meses después de la CB. Sin embargo, no hubo diferencias significativas en el peso perdido entre los intervenidos quirúrgicamente con TPA comparados con los que no presentaban éste, tampoco las hubo entre aquellos que aún presentaban atracones y los que reportaron ya no presentarlos. Los pacientes sometidos a la cirugía con y sin TPA mostraron mejoría significativa en los factores de riesgo cardiopulmonar. Los grupos control reportaron al final del estudio una perdida ponderal de $10.3 \pm 1.5 \%$ con respecto al peso inicial $(P<0.001)$. En comparación, los participantes sometidos a CB con TPA reportaron una pérdida ponderal mayor $(\geq 5 \%$, $p=0.004),(\geq 10 \%, p=0.001),(\geq 20 \%, p=0.001)$. Los pacientes con TPA del grupo quirúrgico reportaron mejoría en los factores de riesgo cardiovascular, evaluados mediante el modelo homeostásico de sensibilidad a la insulina (HOMA) y los niveles de proteína $C$ reactiva, en comparación con el grupo control $(p<0.001)$. Estos datos apoyan la idea de que el TPA no afecta significativamente la pérdida ponderal en los pacientes sometidos a $\mathrm{CB} .^{70}$

\section{Uso de sustancias}

A partir del reporte de un caso que incrementó el consumo de cocaína posterior a la $\mathrm{CB}$, se realizó una revisión sobre consumo de drogas posterior al procedimiento. Es frecuente que los pacientes que acuden para el tratamiento quirúrgico para la obesidad, nieguen o minimicen el consumo de sus- tancias. Sin embargo, existen reportes sobre el consumo de alcohol en el $50 \%$ de los obesos, posicionándose en tercer lugar después del trastorno depresivo y del trastorno de pánico. Por otra parte, entre 2-6\% de los sujetos ingresados en clínicas de adicciones tienen historial de CB. Los factores de riesgo identificados en un estudio para el incremento en el consumo de alcohol fueron: el género masculino, la historia previa de problemas por consumo de alcohol, la menor edad y haber sido sometido a una intervención en $\mathrm{Y}$ de Roux. ${ }^{71}$

Un estudio prospectivo realizado con la hipótesis de que después de la CB los sujetos experimentarían un incremento en el uso de sustancias (alcohol, tabaco y otras drogas), encontró que éste disminuyó el mes posterior a la $\mathrm{CB}$ $(p<0.001)$, cesó la disminución en el tercer mes y, posteriormente, se incrementó de manera paulatina hasta los dos años. $^{72}$

Se ha propuesto que los cambios anatomo-funcionales resultado de la $\mathrm{CB}$ facilitan un aumento en la velocidad de absorción del alcohol, una disminución del tiempo necesario para alcanzar concentraciones séricas máximas, un incremento en estas concentraciones y un mayor tiempo de depuración de éste. Es posible que la disminución del volumen gástrico se asocie con una menor concentración de la enzima alcohol deshidrogenasa y si los niveles de consumo previos a la cirugía se mantienen, la disminución en el IMC tendría algún impacto en la concentración sérica de alcohol. Es decir, un menor IMC para la misma cantidad de alcohol ingerida. Adicionalmente, la disminución en la absorción de vitamina B12 puede significar un riesgo agregado. ${ }^{72}$

Un estudio realizado con la hipótesis alternativa de que un subgrupo de sujetos con consumo de alto riesgo de alcohol (CAR), presentarían una disminución del consumo después de la CB, utilizando los datos del The Assessment of Bariatric Surgery Study (ABS), encontró en los sujetos sometidos a CB una prevalencia del $19 \%$ de CAR y persistió en el seguimiento $17 \%$ al año y $13 \%$ a los dos años $(p=0.01)$. Se reportó mayor disminución en el riesgo de consumo en aquellos sometidos a by pass gástrico comparados con banda gástrica, pero esta diferencia no fue significativa al año de seguimiento y desapareció a los dos años. Un 7\% de los sujetos sin CAR en la medición inicial lo reportaron en el seguimiento a un año y un 6\% lo reportaron en la evaluación a los dos años; sin embargo, la mayoría reportó mejoría en su patrón de consumo. ${ }^{73}$ Estos resultados deben ser tomados con cautela ya que es necesario considerar que además de la cirugía per se, diversos factores contribuyen a la reducción del consumo de alcohol como los programas de acompañamiento postquirúrgico.

Respecto al uso de la cannabis, una revisión realizada con el objetivo de proponer guías preliminares para su abordaje en población bariátrica, no obtuvo información sobre el uso previo y los resultados después de la CB. Aunque, especifica que el consumo de ésta se asoció con efectos nocivos en el sistema respiratorio, cardiovascular y en el Sistema 
Nervioso Central, por lo que los riesgos de complicaciones son mayores para quienes consumen dosis mayores y por tiempos prolongados. ${ }^{74}$

Finalmente, de acuerdo con el National Institute of Health, el abuso de cualquier sustancia es una contraindicación para la CB. Además de lo referido al consumo de alcohol, el tabaquismo se ha asociado con un incremento en el riesgo de muerte en pacientes bariátricos, así como con complicaciones postquirúrgicas. Existe evidencia de que la cocaína puede causar accidentes vasculares cerebrales isquémicos y hemorrágicos en pacientes bariátricos. ${ }^{75}$

\section{Rasgos de personalidad}

La asociación personalidad-obesidad ha sido estudiada debido al impacto de ésta en los aspectos cognitivos, conductuales y psicofisiológicos que contribuyen al desarrollo y mantenimiento del problema entre las personas obesas. ${ }^{76}$ Los principales aspectos cognitivos que favorecen el incremento de peso son los relacionados con la toma de decisiones en torno a la salud y la evaluación de las conductas de riesgo. ${ }^{77}$ Los aspectos conductuales predominantes son la ingesta de alimentos y la falta de ejercicio físico y los aspectos psicofisiológicos descritos hacen referencia a las habilidades de afrontamiento al estrés, las cuales están vinculadas con la actividad del eje hipotalámico-hipofisario-adrenal y con la grasa visceral. ${ }^{78-80}$

El constructo personalidad, ha sido estudiado desde diferentes perspectivas y modelos, lo cual dificulta homogeneizar los resultados de las investigaciones que pretenden estudiar la relación personalidad-obesidad. Existen revisiones sistemáticas dirigidas a agrupar las líneas establecidas en torno a las dimensiones de la personalidad propuestas por algunos modelos similares. Por ejemplo, una revisión que analizó 70 estudios en obesidad y personalidad utilizando el modelo psicobiológico de Cloninger y el modelo de los cinco factores (extroversión, neuroticismo, agradabilidad, concientización y apertura a la experiencia) de la personalidad (FFM, por sus siglas en inglés) encontró que en sujetos obesos predominan la dimensión de neuroticismo y la impulsividad con las respuestas mediadoras de restricción alimentaria e ingesta emocional ante señales externas. La dimensión extroversión y la cooperación fueron asociadas con el sobrepeso, aunque se discute la posibilidad de que las personas con éstas hayan buscado tratamiento y, por lo tanto, afectado la asociación. La sensibilidad a la recompensa se asoció con el incremento ponderal entre las personas con peso normal y con sobrepeso, aunque no con los obesos. ${ }^{76}$ La dimensión agradabilidad y la evitación son controvertidas ya que el estudio previo las correlacionó con obesidad, mientras que otros no encontraron ninguna asociación. ${ }^{78}$ Otro estudio identificó la dimensión concientización como protectora para el sobrepeso y la obesidad, al definir ésta como la capacidad de tomar decisiones sobre impulsos in- ternos, se vinculó con el autocontrol y con la regulación cognitiva de la ingesta. ${ }^{78}$

Resultan interesantes los estudios en población obesa que correlacionaron las variables sociodemográficas, particularmente género y nacionalidad, con las características de personalidad. Un meta-análisis ( $N=78931)$ integró nueve estudios de cohorte que utilizaron el FFM y las variables sociodemográficas, identificando una fuerte asociación entre la dimensión concientización y el sexo femenino, mientras que la extroversión se asoció con el sexo masculino y con los europeos. Al igual que un estudio previo, este metaanálisis identificó sólo a la concientización como dimensión protectora de la obesidad. ${ }^{81}$ Estos resultados coinciden con otros estudios en la asociación de esta dimensión con conductas saludables, como la adherencia a medicamentos, realización de ejercicio físico y pautas de alimentación saludables. ${ }^{78}$

Un estudio realizado con el objetivo de identificar los subtipos de personalidad, utilizando el FFM, y su asociación con psicopatología, trastornos de la alimentación y estilos de afrontamiento desadaptativos en una muestra de 102 pacientes femeninas candidatas a $C B$, sugirió que existen dos subtipos de personalidad, uno bajo en neuroticismo y alto en extroversión, apertura a la experiencia, concientización y agradabilidad y, el segundo, con un perfil opuesto. El primer subtipo fue considerado como resiliente, mientras que el segundo fue considerado emocionalmente inestable o descontrolado. La prevalencia del segundo subtipo fue mayor $(56.9 \%)$ y se asoció con mayor psicopatología, atracones, estilos de afrontamiento maladaptativos y peor pronóstico de la intervención. ${ }^{82}$

\section{DISCUSIÓN Y CONCLUSIÓN}

Hace algunos años no existía un consenso sobre el impacto de los trastornos psiquiátricos antes de la cirugía y la posterior pérdida de peso. Sin embargo, en la última década, las guías de práctica clínica internacionales sugieren detectar la presencia de depresión y de trastornos de la alimentación, en los candidatos a CB. ${ }^{16-19}$ Los estudios incluidos en esta revisión identificaron, además, otros trastornos asociados con la obesidad. Los resultados coinciden en la alta prevalencia de éstos al compararlos con la población general, aunque para algunos trastornos no se encontraron prevalencias específicas en población bariátrica. Debemos considerar que, un obstáculo en la evaluación de los pacientes en protocolo para CB es su percepción del posible riesgo de ser rechazados, por lo que niegan la presencia de psicopatología. Los estudios sobre la asociación TD-obesidad coinciden en los factores asociados al TD en esta población. Además se reportó que el tratamiento de los síntomas depresivos-ansiosos durante la intervención, sea mediante tratamiento quirúrgico o convencional, favorece la optimización de otras intervenciones como una mayor adherencia al régimen de 
alimentación, una mayor motivación para implementar la actividad física y en general contribuye a una mayor pérdida de peso.

Se recomienda no subestimar la asociación TDAH-obesidad y la reportada del primero con TPA, TD y trastorno de ansiedad. Aunque existen pocos estudios sobre el tratamiento farmacológico del TDAH y su impacto en la pérdida de peso, los encontrados en esta revisión reportaron un impacto importante.

Los reportes sobre los trastornos de la conducta alimentaria en pacientes post-operados de $\mathrm{CB}$, proporcionan un dato relevante si se retira el criterio diagnóstico "una gran cantidad de alimentos" un mayor número de sujetos postquirúrgicos cumplirían con el diagnóstico de TPA, dando relevancia al constructo "pérdida de control" como elemento clave de la psicopatología. Sin embargo, aunque los pacientes consideraron su conducta como un atracón, al no cumplir este criterio por sentirse limitados en la ingesta (resultado de la reducción gástrica), no fueron reportados con dicho trastorno. Se recomienda considerar también que otras conductas pueden surgir para compensar el atracón y contribuir a una ingesta calórica excesiva. Entre las preguntas por resolver en investigaciones posteriores se plantean las siguientes: ¿por qué algunos sujetos experimentan reemergencia de los síntomas de atracón, trastorno por atracón y pérdida de control y otros no? ¿cuáles son los factores predictivos de emergencia o re-emergencia de estas conductas? ¿su presencia en el preoperatorio facilita la aparición de otras conductas alimentarias desadaptativas como grazing, que impactan en el resultado ponderal, o bien, estas conductas se sobreponen a los atracones, a la pérdida de control y al trastorno por atracón, generando un mayor impacto sobre la pérdida o ganancia de peso? La variabilidad en los resultados de la CB es muy amplia, sobre todo a largo plazo y las conductas alimentarias desadaptativas pueden contribuir a la recuperación de peso.

Aunque los resultados apoyan la idea de que el TPA no afecta significativamente la pérdida ponderal en los pacientes sometidos a $\mathrm{CB}$, esto no omite el tratamiento del mismo; el estudio que no reportó diferencias en el peso entre pacientes con y sin TPA, había proporcionado tratamiento específico prequirúrgico a aquellos sujetos en los que se identificó el trastorno.

Aunque la $\mathrm{CB}$ está contraindicada en pacientes con abuso de sustancias y existen pocos estudios al respecto, éstos coinciden en una alta prevalencia principalmente en el consumo de alcohol y se evidenció que los autorreportes para identificar su consumo no fueron efectivos.

Es controversial si existe asociación entre el procedimiento quirúrgico y el incremento o disminución en el consumo de sustancias. Los estudios proporcionan resultados contradictorios y algunos reportes presentan limitaciones importantes, por lo que el impacto de la CB en este grupo de pacientes continúa siendo estudiado.
Se encontró que la mayoría de los estudios sobre la asociación personalidad-obesidad utilizaron el FFM. El planteamiento de dos perfiles de personalidad basados en estas dimensiones y los resultados del tratamiento quirúrgico, deben ser realizados también en población masculina y se debe profundizar en investigaciones futuras si uno de estos perfiles puede ser predictivo en el tratamiento de la obesidad.

Finalmente, la gran heterogeneidad de algunos resultados reportados en los estudios puede ser atribuible a varios factores como el tipo de intervención quirúrgica realizada, el tipo de evaluación diagnóstica (entrevistas estructuradas vs. autorreportes) y el sesgo de memoria para evaluar conductas relacionadas con patrones de alimentación.

De los resultados de esta revisión podemos concluir que se ha reportado una alta prevalencia de psicopatología en el paciente que acude para el tratamiento quirúrgico de la obesidad. La presencia de psicopatología impacta en la evolución de la intervención quirúrgica, por lo que el manejo interdisciplinario del paciente obeso que integra a los profesionistas en salud mental, favorece la selección adecuada de los pacientes, la identificación y tratamiento de los trastornos referidos en esta población y, en consecuencia, un mayor éxito en el procedimiento quirúrgico.

\section{Financiamiento}

Ninguno.

\section{Conflicto de intereses}

Los autores declaran no tener ningún conflicto de intereses.

\section{REFERENCIAS}

1. World Health Organization. [Sede Web] Obesity and overweight fact sheet no 311. 2015; [marzo 2016]. http://www.who.int/mediacentre/ factsheets/fs311/en/.

2. Bray GA, Frühbeck G, Ryan DH, Wilding JP. Management of obesity. Lancet 2016. doi:10.1016/S0140-6736(16)00271-3

3. Aguilar-Salinas CA. Non communicable chronic diseases, the main health problem in Mexico. Salud Publica Mex 2013;55(suppl 2):347-350.

4. Barquera S, Campos-Nonato I, Hernández-Barrera L, Pedroza A et al. Prevalence of obesity in Mexican adults 2000-2012. Salud Publica Mex 2013;55(suppl. 2):151-160.

5. Wang Y, Wu Y, Wilson RF et al. Childhood obesity prevention programs: comparative effectiveness review and meta-analysis. Comparative effectiveness review. AHRQ 2013;115.

6. Christian JG, Tsai AG, Bessesen DH. Interpreting weight losses from lifestyle modification trials: using categorical data. Int J Obes 2010;34(1):207-209.

7. Patel D. Pharmacotherapy for the management of obesity. Metabolism. 2015;64(11):1376-1385.

8. Héctor RT. El papel de las opciones quirúrgicas de tratamiento. En: Juan Ángel Rivera Dommarco MH (eds.). Obesidad en México: recomendaciones para una política de Estado. México: Universidad Nacional Autónoma de México; 2012.393-410.

9. Rivera Dommarco JÁ, Obesidad en México: recomendaciones para una política de Estado. México: Universidad Nacional Autónoma de México; 2012. 
10. Buchwald H Bariatric surgery world-wide 2003. Obes Surg 2004;14(9): 1157-1164.

11. Grothe KB, Psychological assessment and management of the weight loss surgery patient. Am J Med Sci 2006;331(4):201-206.

12. Carpiniello B. Obesity and psychopathology. A study of psychiatric comorbidity among patients attending a specialist obesity unit. Epidemiol Psichiatr Soc 2009;18(2):119-127.

13. Scott KM. Obesity and mental disorders in the general population: results from the world mental health surveys. Int J Obes 2008;32(1):192-200.

14. Walker EA. Adult health status of women with histories of childhood abuse and neglect. Am J Med. 1999; 107: 332-339.

15. Álvarez JPJ. The relationship between child abuse and obesty among California women. Am J Prev Med 2007;33:28-33.

16. Fried MHV. Interdisciplinary European guidelines on surgery of severe obesity. Obes Facts 2008;1:52-59.

17. Mechanick JKR. American Association of Clinical Endocrinologists, The Obesity Society, and American Society for Metabolic \& Bariatric Surgery Medical guidelines. Surg Obes Relat Dis 2008;4:109-184.

18. Pull CB. Current psychological assessment practices in obesity surgery programs: what to assess and why. Curr Opin Psychiatry. 2010;23:30-36.

19. LeMont DMM. Suggestions for the presurgical psychological assessment of bariatric surgery candidates. American Society Bariatric Surgery 2004;1-29.

20. Tsuda SBL. Factors affecting rejection of bariatric patients from an academic weight loss program. Surg Obes Relat Dis 2009;5:199-202.

21. Medina-Mora ME. Prevalencia de trastornos mentales y uso de servicios: Resultados de la Encuesta Mexicana de Epidemiología Psiquiátrica en México. Salud Ment 2003;26(4):1-16.

22. Santoncini CU. Determinantes Psicosociales del sobrepeso y la obesidad. En: Juan Ángel Rivera Dommarco MH (ed.). Obesidad en México: recomendaciones para una política de Estado. México: Universidad Nacional Autónoma de México; 2012; pp.189-209.

23. Legenbauer T, de Zwaan M, Benecke A, Mühlhans B et al. Depression and anxiety: Their predictive function for weight loss in obese individuals. Obes Facts 2009;2:227-234.

24. Rutters FNA-P. Acute stress-related changes in eating in the absence of hunger. Obes 2009;17(1):72-77.

25. Ma JXL. Obesity and depression in US women: results from the 20052006 National Health and Nutritional Examination Survey. Obes 2010;18(2):343-353.

26. Eisenberg ME. Associations of weight-based teasing and emotional well-being among adolescents. Arch Pediatr Adolesc Med 2003;157:733-788.

27. Murphy JM. Obesity and weight gain in relation to depression: findings from the Stirling Country Study. Int J Obes 2009;33(3):335-341.

28. Mukamal KJ. BMI and risk factors for suicide: why is BMI inversely related to suicide? Obes 2009;17(3):532-538.

29. Bhatti JA, Nathens AB, Thiruchelvam $D$, Grantcharov $T$ et al. Selfharm emergencies after bariatric surgery: a population-based cohort study. JAMA Surg 2016;151(3):226-232. doi:10.10001|jamasurg.2015.3414

30. Knop J, Fisher A. Duodenal ulcer, suicide, psychopatology and alcoholism. Acta Psychiatr Scand 1981;63:346-355.

31. Ostlund MP, Backman $\mathrm{O}$, Marsk et al. Increased admission for alcohol dependence after gastric bypass surgery compared with restrictive bariatric surgery. JAMA Surg 2013;148:374-377.

32. Mitchell JE, Crosby R, de Zwaan $M$ et al. Possible risk factors for increased suicide following bariatric surgery. Obes (Silver Spring) 2013;21:665-672.

33. Andrews ZB. The extra-hypothalamic actions of ghrelin on neuronal function. Trensa Neurosci 2011;34: 31-40.

34. Altfas JR. Prevalence of attention deficit/hyperactivity disorder among adultd in obesity treatment. BMC Psychiatry 2002;2(9):1-8. doi:10.81186 |1471-244x-2-9

35. Samuele Cortese MA. Obesity in men with childhood ADHD: A 33-year controlled, prospective, follow-up Study. Pediatrics 2013. doi:10.1542/peds.2012-0540

36. Cortese S. Obesity and ADHD: Clinical and neurobiological implications. Curr top Behav Neurosci doi:10.1007/7854_2011_154,

37. Agranat-Meged A. Attention deficit hyperactivity disorder in obese melanocortin-4-receptor (MC4R) deficient subjects: a newly described expression of MC4R deficiency. Am J Med Genet B Neuropsychiatr Genet 2008;15:47-53.

38. Albayrak Ö. Successful methylphenidate treatment of early onset extreme obesity in a child with a melanocortin-4 receptor gene mutation and attention deficit/hyperactivity disorder. Eur J Pharmacol 2011;660(1):165-170.

39. Pott W. Successful treatment with atomoxetine of adolescent boy with attention/deficit/hyperactivity disorder, extreme obesity, and reduced melanocortin-4-receptor function. Obes Facts 2013;6(1):109115.

40. Faraone SV. Long-term effects of extended-release mixed amphetamine salts treatment of attention-deficit/hyperactivity disorder on growth. J Child Adolesc Psychopharmacol 2005;15:191-202.

41. Volkow ND. Reward, dopamine and the control of food intake: implications for obesity. Trends Cogn Sci 2011;15(1):37-46.

42. Davis C. Psychobiological traits in the risk profile for overeating and weight gain. Int J Obe. 2009;33:49-53.

43. Davis C. A psycho-genetic study of associations between the symptoms of binge eating disorder and those of attention deficit (hyperactivity) disorder. J Psychiatr Res 2009;43(7):687-696.

44. Seitz J. The role of impulsivity, inattention and comorbid ADHD in patients with bulimia nervosa. PLoS One 2013;20(8):1-8 e63891.

45. Docet MF. Attention deficit hyperactivity disorder increases the risk of having abnormal eating behaviours in obese adults. Eat Weight 2012;17(2):132-136.

46. de Zwaan M. Binge eating disorder and obesity. Int J Obes Suppl 2001;25:S51-S55.

47. Altfas JR. Prevalence of attention deficit/hyperactivity disorder among adults in obesity treatment. BMC Psychiatry 2009:13.

48. Alfonsson S. Screening of adult ADHD among patients presenting for bariatric surgery. Obes Surg 2012; 22(6):918-926.

49. Goodman DW. The consequences of attention-deficit/hyperactivity disorder in adults. J Psychiatr Pract 2007;13:318-327.

50. Sven Alfonsson TP. Screening of adult ADHD among patients presenting for bariatric surgery. Obes Surg 2012;22:918-926.

51. Karlsson J. Ten-year trends in health-related quality of life after surgical and conventional treatment for severe obesity: the SOS intervention study. Int J Obes 2007;31:1248-1261.

52. Burgmer RI. Psychological outcome two years after restrictive bariatric surgery. Obes Surg 2007;17:785-791.

53. Levy LD. Treatment of refractory obesity in severely obese adults following management of newly diagnosed attention deficit hyperactivity disorder. Int J Obes 2009;33(3):326-334.

54. Carlos M. Grilo. Binge eating and self-esteem predict body image dissatisfaction among obese men and women seeking bariatric surgery. Int J Eat Disord 2005;37:347-351.

55. Monteleone P. Dysfunctions of leptin, ghrelin, BDNF and endocannabinoids in eating disorders: Beyond the homeostatic control of food intake. Psychoneuroendocrinology 2013;38:312-330.

56. Palma-Coca O. Association of socioeconomic status, problem behaviors, and disordered eating in Mexican adolescent: Results of the Mexican National Health and Nutrition Survey 2006. J Adoles Health 2011;49(4):400-406.

57. Psiquiatría AA. Manual Diagnóstico y Estadístico de los Trastornos Mentales DSM V. Washington: Arlington; 2014.

58. Niego HS. Binge eating in the bariatric surgery population: A review of the literature. Int J Eat Disord 2007;40:349-359.

59. Hsu LK. Eating disturbances before and after vertical banded gastroplasty: A pilot study. Int J Eat Disord 1996;19:23-34. 
60. Lang T. Impact of gastric banding on eating behavior and weight. Obes Surg 2002;12:100-107.

61. Mitchell JE, Hatsukami D, Eckert ED, Pyle RL. Eating disorders questionnaire. Psychopharmacol Bull 1985;21:1025-1043.

62. Saunders R. Binge eating in gastric bypass patients before surgery. Obes Surg 1999;9:72-76.

63. Kalarchian MA. Binge eating among gastric bypass patients at longterm follow-up. Obes Surg 2002; 12:270-275.

64. Busetto L, Segato G, De LM et al. Weight loss and postoperative complications in morbidly obese patients with binge eating disorder treated by laparoscopic adjustable gastric banding. Obes Surg 2005;15:195201.

65. LaShanda RJC. Axis i psychopathology in bariatric surgery candidates with and without binge eating disorder: Results of structured clinical interviews. Obes Surg 2012;22(3):389-397.

66. De Zwaan M. Binge eating disorder. Ther Umsch 2016;63(8):529-533.

67. Faiburn CG. Risk factors for binge eating disorder: a community-based, case-control study. Arch Gen Psychiatry 1998;55(5):425-432.

68. Conceicão E. Eating disorders after bariatric surgery: A case series. Int J Eat Disord 2013;46:274-279.

69. Kofman MD. Maladaptive eating patterns, quality of life, and weight outcomes following gastric bypass: Results of an Internet survey. Obes 2010;18:1938-1943.

70. Wadden TA. Binge eating disorder and the outcome of bariatric surgery at one year: A prospective, observational study. Obesity (Silver Spring) 2011;19(6):1220-1228.

71. Quevedo Y. Cambio en el patrón de consumo de sustancias posterior a cirugía bariátrica: presentación de un caso clínico. Rev Med 2015;143:116-119.
72. Conason A. Substance use following bariatric weight loss surgery. JAMA Surg 2013;148(2):145-150.

73. Wee $\mathrm{ChC}$. High risk alcohol use after weight loss surgery. Surg Obes Relat Dis 2014;10(3):508-513.

74. Rummell ChM. Assessing marijuana use in bariatric surgery candidates: Should it be a contraindication? Obes Surg 2014;24:1764-1770.

75. Choy JY, Scarborough TK. Stroke and seizure following a recent laparoscopic Roux-en- Y gastric bypass. Obes Surg 2004;14:857-860.

76. Gerlach G, Herpetz S, Loeber S. Personality traits and obesity: a systematic review. Obes Rev 2015;16:32-63.

77. Flynn KE, Smith MA. Personality and health care decision-making style. J Gerontol B Psychol Sci Soc Sci 2007;62:261-267.

78. Bogg T, Roberts BW. Conscientiousness and health-related behavior: a meta-analysis of the leading behavioral contributors to mortality. Psychol Bull 2004;130:887-919.

79. Caspi A, Roberts BW. Personality development across the life course: the argument for change and continuity. Psychol Inq 2001;12:49-66.

80. Magiakou MA, Chrousos GP. Biological basis of stress-related diseases. En: Antoniou ASG, Cooper CL (eds.). Research companion to organizational health psychology. Cheltenham: Edward Elgar; 2005; pp.70-86.

81. Jokela M, Hintsanen M, Hakulinen C et al. Association of personality with the development and persistence of obesity: a meta-analysis based on individual-participant data. Obes Rev 2013;14:315-323.

82. Claes L, Vandereycken W, Vandeputte A, Braet C. Personality subtypes in female pre-bariatric obese patients: do they differ in eating disorder symptoms, psychological complaints and coping behavior? Eur Eat Disord Rev 2013;21:72-77. 